Mens

revue d'histoire intellectuelle de l'Amérique française

\title{
Le voyage identitaire (et imaginaire) de Tocqueville au Bas-Canada : vieille France ou Nouvelle-France?
}

\section{Anne Trépanier}

Volume 5, numéro 1, automne 2004

URI : https://id.erudit.org/iderudit/1024390ar

DOI : https://doi.org/10.7202/1024390ar

Aller au sommaire du numéro

Éditeur(s)

Centre de recherche en civilisation canadienne-française

ISSN

1492-8647 (imprimé)

1927-9299 (numérique)

Découvrir la revue

Citer cet article

Trépanier, A. (2004). Le voyage identitaire (et imaginaire) de Tocqueville au Bas-Canada : vieille France ou Nouvelle-France ? Mens, 5(1), 119-149. https://doi.org/10.7202/1024390ar
Résumé de l'article

Guidée par un questionnement sur la part imaginaire de l'identité, nous abordons le voyage de Tocqueville au Bas-Canada comme une excursion à la recherche du Même. À une réflexion sur la différence entre les Canadiens français et les Français, sur leur apparente ressemblance, sur le contraste du rapport des Canadiens français avec les Américains et les Anglais et, dans une moindre mesure, avec les Français, succède une prise de position marquée de Tocqueville pour l'affirmation du fait français au Canada. Dans ce pays qu'il considère comme une nouvelle vieille France, le jeune aristocrate brosse un tableau clair-obscur des Canadiens, réjouissantes momies de l'ancienne France conservées dans le musée imaginaire de l'identité. 


\title{
LE VOYAGE IDENTITAIRE (ET IMAGINAIRE) DE TOCQUEVILLE AU BAS-CANADA : VIEILLE FRANCE OU NOUVELLE-FRANCE ?
}

\author{
Anne Trépanier \\ Département d'histoire \\ Université Laval
}

\section{Résumé}

Guidée par un questionnement sur la part imaginaire de l'identité, nous abordons le voyage de Tocqueville au Bas-Canada comme une excursion à la recherche du Même. À une réflexion sur la différence entre les Canadiens français et les Français, sur leur apparente ressemblance, sur le contraste du rapport des Canadiens français avec les Américains et les Anglais et, dans une moindre mesure, avec les Français, succède une prise de position marquée de Tocqueville pour l'affirmation du fait français au Canada. Dans ce pays qu'il considère comme une nouvelle vieille France, le jeune aristocrate brosse un tableau clair-obscur des Canadiens, réjouissantes momies de l'ancienne France conservées dans le musée imaginaire de l'identité.

\section{Abstract}

This article explores Alexis de Tocqueville's journey to Lower Canada in an effort to understand the role of the imaginary in the formation of identity. Tocqueville was fascinated by the differences and similarities that existed between the French and the Canadiens, and he was an ardent supporter of French Canadian survivance. However, the young aristocrat considered Lower $\mathrm{Ca}$ nada to be a relic of the French Ancien Régime and saw its inbabitants as joyous artefacts in an imagined museum of identity. 
Les voyages ont ceci de prévisible qu'ils mènent ceux qui les font vers des territoires déjà occupés par leur imaginaire. L'imagination précède le voyageur à la façon d'un éclaireur. Les aventuriers parcourant un pays étranger ont ainsi la curieuse sensation de l'avoir vu en rêve ou de s'y être déjà frottés. L'imagination débroussaille, fait des sentiers, annonce le meilleur parcours au voyageur qui laisse s'installer son imaginaire avant lui. S'il est rassurant, c'est en ce qu'il conforte les préjugés et les idées reçues. S'il est nécessaire, c'est qu'il apaise les différences criantes, favorise le développement d'éléments de comparaison fondés sur la similitude et, ce faisant, prépare l'échange culturel et facilite les rencontres. De l'autre côté du miroir, les hôtes cherchent, en appelant les compliments des visiteurs sur leur accueil et sur leur pays, des éléments de confirmation de leur identité. Identité voulue, projetée, attendue ou vérifiée, la reconnaissance est le défi constant de la représentation et de l'observation.

À la fin de l'été 1831, au cours de leur séjour de dix mois aux États-Unis, Alexis de Tocqueville et Gustave de Beaumont vont se prendre au jeu de l'investigation du territoire imaginaire de la Nouvelle-France en visitant la curieuse colonie britannique qui fut jadis possession française. Partis officiellement en Amérique afin d'étudier le système pénitentiaire des États-Unis, ils mènent parallèlement une enquête d'envergure sur le phénomène démocratique de la jeune république. Tocqueville et Beaumont se prêtent aussi, brièvement, à l'exploration touristique. « Le voyage à la mode ", pour reprendre l'expression de Tocqueville, les amène à admirer les chutes du Niagara. Puis, leur route les conduit au BasCanada. Elle les porte alors vers un voyage, qui les appelle cette fois à visiter le territoire imaginaire de leur identité. Il semble que, pour Tocqueville, cette excursion au Bas-Canada fut non seulement un périple empli de découvertes et em- 
preint d'étonnement, dont les traces narratives sont d'utiles clichés journalistiques de la réalité bas-canadienne en $1831^{1}$, mais encore un voyage dans l'imaginaire de son identité, dont les références territoriales et mentalitaires ${ }^{2}$ immédiates sont celles de la France d'Ancien Régime.

Au-delà des remarques attendues, les notes d'observation de Tocqueville sont un trésor pour ceux qui en sont l'objet ainsi que pour leurs héritiers. Sans compter que ces mêmes documents recèlent des données sur l'identité du voyageur lui-même, de ce qu'il cherche à voir et de ce qui lui apparaît pertinent ou problématique, suivant la route dessinée par son imaginaire. C'est cette piste que nous explorons dans cet article en usant de sources historiques et conceptuelles. Nous employons des commentaires explicites de Tocqueville sur le Bas-Canada et ses habitants. Nous utilisons aussi les notions d'imaginaire et d'identité. Selon Paul Ricœur, l'identité est issue de la dialectique entre les concepts de mêmeté, d'ipséité et d'altérité. La mêmeté réfère aux caractères d'un objet indivisible que le temps laisse perdurer, par exemple, le nom d'un individu. L'ipséité désigne l'aspect de changement de cet objet, par exemple, le vieillissement de ce même individu. L'objet indivisible (l'individu) apparait donc constitué de mêmeté et d'ipséité. De plus, il est structuré par l'altérité, c'est-à-dire par son rapport aux autres. L'identité de l'individu est le résultat de toutes ces forces en présence : mêmeté, ipséité, altérité. Nous reprenons cette définition de l'identité puisqu'elle est fondatrice de toute la recherche actuelle sur la problématique de l'identité narrative dans le récit historien mais aussi, dans le récit historique comme celui de Tocqueville. 


\section{Les sources}

Il est impossible de comparer en étendue et en profondeur, en termes de rigueur méthodologique et de qualité de l'information, les observations canadiennes de Tocqueville à ses études américaines ${ }^{4}$. D'abord, les dix-huit mois de permission qu'ont obtenus Tocqueville et Beaumont sont, de façon prioritaire, consacrés à l'étude du cas américain. Il en résultera $D u$ système pénitentiaire aux États-Unis et de son application en France puis, pour Tocqueville, De la démocratie en Amérique en deux volumes (1835 et 1840). Beaumont publiera pour sa part un roman à thème: Marie ou l'esclavage (1835).

Les notes concernant le Bas-Canada sont d'un tout autre ordre puisqu'elles correspondent à des impressions rédigées sur le vif pendant un court voyage. On ne saurait trop insister sur le caractère spontané des observations et des brèves réflexions qui y sont compilées. Tocqueville a cependant mené une enquête sérieuse balisée par un questionnaire établi qui vaut à son auteur d'être qualifié de "pré-sociologique ». En effet, comme l'a écrit René Rémond, "Tocqueville [a] l'honnêteté, qui n'est pas si fréquente à l'époque, de citer ses sour$\operatorname{ces}^{5} »$. Bien que ses notes soient enchevêtrées, ses interlocuteurs sont bien identifiés, de même que les différents lieux où prennent place ses réflexions. Le nombre d'entretiens réalisés est toutefois trop faible pour conclure à une étude scientifique. Par ailleurs, les notes de Tocqueville sont rassemblées dans différents cahiers contenant des impressions, des fiches, des bilans provisoires et, par moments, des récits reprenant des conversations ou des observations. Seulement, ces cahiers sont difficiles à regrouper ${ }^{6}$. Cette imbrication de notes témoigne de l'absence d'une vision d'ensemble ou d'une narration cohérente et soutenue pouvant émaner de son séjour au Bas-Canada. 
Les informations recensées par Tocqueville auraient certainement gagné à être confrontées à celles contenues dans le journal de Beaumont. Celui-ci fut malheureusement perdu, à notre connaissance, à l'exception de quelques extraits que nous retrouvons compilés avec sa correspondance personnelle, cités chez d'autres auteurs, recensés par André Jardin et Georges Pierson, et repris par Claude Corbo dans son recueil de textes Regards sur le Bas-Canada. Hormis Quinze jours dans le désert, aucun des textes écrits par Tocqueville au cours de la période qui nous intéresse n'était destiné à la publication? Ainsi, les critiques que nous apportons visent seulement à rétablir l'importance de certains passages en vue de notre brève étude sur le voyage identitaire de Tocqueville au Bas-Canada et ne souligne en aucun cas l'absence de références exhaustives sur tel ou tel aspect de la vie de la colonie, ce que l'historiographie canadienne et québécoise déplore. « Heureusement, remarque Corbo, la perspicacité, la clairvoyance, la sagacité remarquables de Tocqueville purent compenser la brièveté de son temps d'observation sur place ${ }^{8}$.»

Il faut imaginer l'esprit dans lequel les deux amis sont venus au Canada. En août 1831, les objectifs de Tocqueville se résument dans la volonté d'observer l'état de l'opinion publique au Canada sur la situation ethnique, politique, sociale et religieuse dans la colonie. Pour ce faire, il procède à l'établissement d'une documentation puis à la vérification des renseignements obtenus de différents acteurs sociaux en croisant leurs réponses à un même questionnaire". Dans ses carnets non-alphabétiques 1,2 et 3 , Tocqueville reconstitue ses entretiens sous forme de conversation. Les questions préliminaires sont susceptibles d'être subdivisées, comme le laissent croire des entrevues plus importantes avec des Canadiens «immigrés ». C’est le cas de John Neilson, député de Québec d'origine écossaise, et du père Quiblier, né en France 
et ordonné à Grenoble, directeur du Séminaire sulpicien de Montréal. Les entretiens s'ouvrent sur des questions d'ordre numérique ou statistique, mais ils sont organisés de manière à aller rapidement vers des questions plus difficiles concernant la situation politique. Par exemple, sur la question ethnique, Tocqueville demande d'abord " quelles sont les proportions respectives des deux populations ». Il poursuit par une deuxième question plus inquisitrice : "Quelle est la nature des rapports entre les deux communautés ?». Il enchaîne enfin par une troisième question, clairement provocatrice : "peut-on envisager l'éventualité d'un soulèvement canadienfrançais ? ». Parfois, des interrogations mineures sont intercalées au questionnaire type. Considérant l'élan rhétorique de l'interlocuteur ou son éventuel malaise, Tocqueville va au bout de son questionnaire en prenant des notes précises, dépassant la transcription des seules réponses verbales, pour décrire avec le plus de netteté possible l'impression se dégageant d'une mimique ou d'un geste nerveux. Ainsi en est-il lors de l'entrevue de Nomen Blanc, négociant anglais à Québec :

En parlant des Canadiens, il se peignait sur la physionomie flegmatique [de Monsieur Blanc] un sentiment de haine et de mépris très visible. Il est rare qu'on parle avec tant de passion de ceux dont on ne redoute rien ${ }^{10}$.

De même, lors de son entrevue avec John Neilson, il dit avoir posé sa question (" pensez-vous que la race française parvienne à jamais à se débarrasser de la race anglaise ?") " avec précaution, attendu la naissance de l'auteur ${ }^{11}$ ». La qualité d'Écossais de naissance de Neilson donne du charme à cet entretien et, selon Tocqueville, témoigne de la générosité politique des Canadiens. En effet, Tocqueville s'enthousiasme du fait que les Canadiens accueillent, dans leur combat politique, un représentant à la Chambre d'Assemblée qui soit d'une 
autre culture et d'une autre foi que la majorité d'entre eux ${ }^{12}$. L'adhésion de John Neilson au Parti canadien en tant que " réformiste modéré » aurait d'ailleurs apporté "une caution morale essentielle » à cette organisation politique qui veut redéfinir l'identité canadienne en mettant de l'avant les institutions libérales. Admiratif des institutions britanniques et des institutions municipales américaines, diffuseur de la philosophie des Lumières, le député John Neilson est un interlocuteur de choix pour Tocqueville, qui devise longuement avec l'imprimeur-éditeur et libraire le plus puissant du Bas-Canada.

Les principales critiques documentalistes font cependant état du petit nombre de personnages publics d'envergure interrogés par Tocqueville, qui ne s'était pas prévalu de sa qualité de fonctionnaire français en mission pour solliciter un entretien avec le gouverneur Aylmer ni avec le chef du Parti patriote, Louis-Joseph Papineau ${ }^{13}$. Ce sont là les fameuses « rencontres manquées » remarquées par l'historiographie canadienne, qui causent d'ailleurs une inquiétude à Tocqueville :

Je n'ai encore vu au Canada aucun homme de talent, ni lu une production qui en fit preuve. Celui qui doit remuer la population française et la lever contre les Anglais n'est pas encore né [...]. Mais parviendront-ils jamais à reconquérir complètement leur nationalité ? C'est ce qui est probable sans malheureusement être assuré. Un homme de génie qui comprendrait, sentirait et serait capable de développer les passions nationales du peuple aurait ici un admirable rôle à jouer. Il deviendrait bientôt l'homme le plus puissant de la colonie. Mais je ne le vois nulle part ${ }^{14}$.

Munis d'une lettre d'introduction écrite de la main de John Canfield Spencer, légiste bien en vue et ami de fraîche date rencontré aux États-Unis ${ }^{15}$, Tocqueville et Beaumont rencontrent les frères Dominique et Charles Mondelet, avo- 
cats à Montréal. De même, le révérend John Power de New York ouvre à Tocqueville la porte du Séminaire de Montréal par une lettre adressée au père Quiblier. Ces rencontres sont certes privilégiées par les contacts aristocratiques de Tocqueville, mais les suivantes sont davantage provoquées par son scrupule empiriste d'honnête homme. Les entrevues demandées à John Neilson et au conseiller législatif Denis-Benjamin Viger, cousin de $M^{\text {st }}$ Lartigue et de Louis-Joseph Papineau, ainsi qu'au juge Jean-Thomas Taschereau, sont de cet ordre. Tocqueville narre: "Nous nous sommes écartés dans les sentiers et nous avons causé avec tous les habitants que nous avons rencontrés, tâchant de faire porter la conversation sur des sujets graves ${ }^{16}$. $\gg$ Toutefois, selon l'avis de JeanMichel Leclercq, auteur du premier mémoire d'historiographie critique portant sur Tocqueville au Canada, ceux qu'il rencontre ne sont guère représentatifs de la diversité de l'opinion générale :

pour le clergé, un prêtre français [Quiblier], et pour le personnel politique, un député qui sera, l'année suivante, exclu de son parti et déclaré «traitre à sa race » [D. Mondelet] et un chef politique dont l'influence diminue graduellement et qui se retirera trois ans plus tard [. Neilson $]^{17}$.

Les personnalités interrogées par Tocqueville représentent toutefois, selon le politologue Stéphane Dion, "des interlocuteurs de tendance modérée, militant pour l'application effective de l'Acte constitutionnel de $1791^{18}$ ", c'est-à-dire défendant un gouvernement représentatif dans la tradition parlementaire de Londres. Loin d'être les doubles canadiens des anciens Français, ces Canadiens, conservateurs à certains égards, sont néanmoins inspirés par le modèle américain. $\mathrm{Si}$ Tocqueville note sciemment que : "Les Français d'Amérique sont aux Français de France ce que les Américains sont 
aux Anglais ${ }^{19}$ ", c'est d'abord pour le confort de l'analogie à son œil de voyageur. Il tente de replacer ses repères. Il ne réussit cependant pas à identifier comme tel ce vecteur de différence mentalitaire qu'est le fait de vivre sur le territoire américain en adoptant une mentalité différente. Tocqueville se borne à conclure «En un mot ils ont en eux tout ce qu'il faudrait pour créer un grand souvenir de la France dans le Nouveau Monde. »

\section{La quête du Même}

La France du XIX ${ }^{e}$ siècle est ouverte sur le monde, mais elle a perdu sa colonie de la vallée du Saint-Laurent depuis le traité de Paris de 1763. Elle n'aurait certes plus «suppos[é] à un Canadien une figure extraordinaire et des mœurs encore plus étranges ", comme le faisait l'éditeur de Pierre Pouchot en 1781 dans sa préface aux Mémoires sur la dernière guerre de l'Amérique septentrionale ${ }^{20}$. La France prêtait toutefois à son ancienne colonie une attitude de repli et, sans doute, craignait pour la survivance du fait français en Amérique, si elle ne le prenait pas déjà pour avili, voire anéanti. Tocqueville témoigne de cet état d'esprit, dans un commentaire glissé dans une lettre à son ancien précepteur, l'abbé Lesueur : «Il n’y a pas six mois, je croyais, comme tout le monde, que le Canada était devenu complètement anglais ${ }^{21}$. " Encore pouvons-nous souligner la fantaisie mêlée de vérité historique de l'auteur du Génie du christianisme (1802) 22 , qui relève des passages colorés référant à la Nouvelle-France jusque dans ses posthumes Mémoires d'outre-tombe (1849-1850). Châteaubriand, racontant son voyage de 1791 à son cousin éloigné, avait peut-être donné la couche de fond à la toile sur laquelle le jeune Tocqueville allait brosser son tableau du Bas-Canada. Aussi, chez les BoisBrûlés, Tocqueville sera-t-il en situation d'intense étrangeté. À la surprise d'entendre un Métis lui adresser la parole en français, il a cette réflexion pour le moins étonnante : «Mon 
cheval m'aurait adressé la parole que je n'aurais pas, je crois, été plus surpris ${ }^{23}$ ! ».Plus tôt, à la hauteur de Sault-Sainte-Marie, il avait griffonné rapidement: "Singulier effet que cause sur nous cette langue française entendue à la fin du monde et avec ses vieilles tournures et son accent provincial : laridondaine, laridondon ${ }^{24}$. " Dans l'intérêt que Tocqueville manifeste pour la mentalité et l'espace canadiens, il y a, nous semble-t-il, une large part de nostalgie de la "vieille nation française » combinée avec l'élan, fantasmatique, d'approcher l'image du Canadien en tant que frère identitaire, de sang et de langue. Le Canada de Tocqueville, résumé à la vallée du fleuve Saint-Laurent où est concentrée la population de langue française, est un lieu doublement visité par Tocqueville. Étrangère et connue à la fois, la contrée canadienne reçoit aussi la visite de l'imaginaire de Tocqueville : «Le Canada est sans comparaison la portion de l'Amérique jusqu'ici visitée par nous, qui a le plus d'analogie avec l'Europe et surtout la France ${ }^{25}$.» À partir des références qui habitent son imaginaire, Tocqueville découvre un Nouveau Monde, en y déployant sa curiosité et son imagination. Il ne reconnait toutefois pas tout à fait ses contemporains Français, lorsqu'il s'attarde à observer les Canadiens comme de «bons enfants» de France.

Arrêtons-nous un moment sur le concept de mêmeté. Nous avons déjà exposé notre vision du voyage, ou de la rencontre, au sens large, selon laquelle la différence est évacuée par l'imaginaire au profit des similitudes. Dans le cas du voyage identitaire, on cherche, consciemment ou non, à trouver du connu dans ce paysage humain et terrestre différent. Or, les observations que fait Tocqueville nous ramènent au concept d'identité. Que cherche-t-il en posant des comparaisons avec la France de l'Ancien Régime? Les différences, le pareil, le même? La mêmeté, pour Paul Ricœur, c'est la référence à l'identique, c'est l'idem. L'exploration de la société bas-cana- 
dienne par Tocqueville est soumise à cette recherche, mais, bien évidemment, ne s'y résume pas. Les commentaires de Tocqueville partent de l'espace connu : historique, appris et vécu. Dans le cas de Tocqueville, dont la famille aristocratique a douloureusement vécu la Révolution française du côté de la monarchie, le fonds identitaire est noyauté autour de la mentalité d'Ancien Régime. Le jeune Alexis est toutefois porté en faveur des nouvelles idées libérales. Corbo explique :

Face à cette cascade de régimes qui oscillent entre l'autoritarisme et le libéralisme [Napoléon Bonaparte, Restauration de 1814, les Cent-Jours de Napoléon en 1815, la nouvelle restauration, la monarchie libérale et constitutionnelle issue de la révolution de juillet 1830], Tocqueville rejette la nostalgie et l'aveuglement politique de nombre de membres de sa classe qui rêvent d'un impossible retour à l'Ancien Régime. Il s'emploie plutôt à comprendre le devenir des sociétés de son temps $[\ldots]^{26}$.

Émerveillé par la démocratie américaine, il voit d'un œil trouble le mélange des héritages sur la terre bas-canadienne. De sa confusion jaillissent des éléments neufs, qui font grincer sa recherche du Même.

La question de la " mêmeté » identitaire entre Canadiens et Français se pose moins explicitement chez un Français voyageur comme Tocqueville que chez un ministre français des Colonies du XVII ${ }^{e}$ siècle comme Jean-Baptiste Colbert. On peut cependant arguer que l'interprétation sociologique que fait Tocqueville est en quête du Même. En effet, l'observateur construit sans cesse ses comparaisons sur le paradigme de la différence. D'une part, il éloigne les mœurs des Canadiens de celles des Américains et des Anglais. D'autre part, il favorise le catalogue des ressemblances perçues ou construites avec la France d'Ancien Régime. La difficulté identitaire 
de Tocqueville se situe précisément sur ce plan. Si les Canadiens ressemblent aux Français, ils sont semblables à ceux d'une France désormais disparue. Aussi, la quête du Même est-elle balisée par un imaginaire décalé du présent canadien par rapport à la contemporanéité française du fonctionnaire en mission aux États-Unis. Ainsi, pour Tocqueville, l'avenir du Canada ne peut-il être, dans une perspective autonomiste par rapport à la Grande-Bretagne, qu'une nouvelle vieille France.

Interrogé par Tocqueville, John Neilson rapporte l'histoire du Canada, en bon précepteur et inspecteur des écoles : «Le Français qui est peut-être le peuple qui garde le plus en définitive sa trace originelle, est cependant celui qui se plie le plus facilement pour un temps aux mœurs, aux idées, aux préjugés de celui chez lequel il vit ${ }^{27}$.» Chez les Canadiens, Tocqueville trouve des similitudes avec les Français d'autrefois; ils sont à la fois fidèles "au trône et à l'autel ${ }^{28}$ ", simples et accueillants : "Les Canadiens nous entourent avec la franchise et la bonhomie des Français ${ }^{29}$. " Plutôt que de les trouver " ensauvagés ", Tocqueville s'émerveille, comme d'autres voyageurs, de "l'authenticité » des Canadiens, comme s'ils étaient destinés à la conservation des mœurs et des coutumes françaises pour un musée du patrimoine vivant. Tocqueville écrit ainsi à son père, depuis une embarcation sur le lac $\mathrm{Hu}$ ron, que les individus qu'il a rencontrés sont pareils aux « Français d'il y a un siècle, conservé[s] comme [des] momie[s] pour l'instruction de la génération actuelle $e^{30} \% . \mathrm{Si}$, selon son appréciation ethnographique, les Canadiens français sont «le plus beau rejeton de la famille européenne dans le NouveauMonde $^{31}$ ", s'ils ont réuni « tous les éléments d'un grand peu$\mathrm{ple}^{32}$ », il demeure toutefois que, pour Tocqueville, ce peuple est aussi menacé culturellement et connait une dépendance économique ainsi qu'une pauvreté qui se traduisent, en ter- 
mes positifs, par la manifestation d'un savoir-faire manuel qui impressionne le jeune aristocrate: «Le paysan canadien fait lui-même tous les objets de nécessité : il fait ses souliers, ses habits, toutes les étoffes de laine qui le couvrent (je l'ai $v u)^{33}$." Il ajoute qu'«il est facile de voir que les Français sont le peuple vaincu. Les classes riches appartiennent pour la plupart à la race anglaise ${ }^{34} \%$.

Il semble que les carnets de voyage de Tocqueville répondent positivement à une curiosité pour le Même français disparu dans la France nouvelle. Ses promenades «sans guide » dans les environs de Québec lui inspirent ses premières remarques sur l'apparence du Canada, empreintes de nostalgie romantique, nourrissant l'imaginaire de la vieille France :

Les villages que nous avons vus aux environs [de Québec] ressemblent extraordinairement à nos plus beaux villages. On n'y parle que le français. La population parait heureuse et aisée. Le sang y est remarquablement plus beau qu'aux États-Unis. La race y est forte, les femmes n'ont pas cet air délicat et maladif qui caractérise la plupart des Américaines ${ }^{35}$.

Il y a dans cet extrait davantage que «le mal du pays» dont aurait pu souffrir Tocqueville. Il s'agit ici d'un paysage réel, mis en opposition avec celui des États-Unis et en rapport direct avec la France. Mais de quelle France s'agit-il ? Bien sûr, les villes et les villages, les vallées et les montagnes sont les mêmes qu'avant la Révolution française. Mais ces personnages qui animent le tableau canadien sont-ils Français? Il semble en effet que cette représentation des Canadiens comme étant des anciens Français soit restée marquée dans son œuvre puisqu'il écrit, dans L'Ancien Régime et la Révolution, que c'est: "un peuple tellement inaltérable dans ses principaux instincts qu'on le reconnait encore dans des portraits qui ont été faits de lui il y a deux ou trois mille ans ${ }^{36}$ \%. 
Dans un cahier portatif, il notait, en se souvenant de son séjour au Bas-Canada :

Si la nature n'a pas donné à chaque peuple un caractère indélébile, il faut avouer du moins que des causes physiques ou politiques ont fait prendre à l'esprit d'un peuple, sont bien difficiles à arracher, même quand il s'agit d'être soumis à aucune de ces causes. Nous avons vu au Canada des Français vivant depuis soixante-dix ans sous le gouvernement anglais, et restés absolument semblables à leurs anciens compatriotes de France. Au milieu d'eux vit une population anglaise qui n'a rien perdu de son caractère national ${ }^{37}$.

À propos des Canadiens français, Tocqueville écrivait: «On ne peut contester leur origine, ils sont aussi Français que vous et $\mathrm{moi}^{38}$ », et encore, à l'abbé Lesueur : « Nous nous sentons chez nous, et partout on nous reçoit en compatriotes, en fils de la vieille France. À mon avis, cette épithète est mal choisie : la vieille France, elle est au Canada, et la nouvelle est chez nous ${ }^{39}$.»

On a vu que la problématique identitaire, chez un Tocqueville observant les Canadiens, est celle du Même. Tocqueville va jusqu'à faire l'amalgame des Canadiens et des anciens Français dans sa lettre à l'abbé Lesueur. S'il a perçu nombre de différences entre la France qui lui est contemporaine et le Bas-Canada, Tocqueville refuse d'imputer des caractéristiques américaines à la mentalité et à la culture canadiennes. Aussi, ce n'est que par les figures repoussoirs des Anglais et des Américains qu'il arrive à définir les Canadiens sans jamais leur opposer les traits de la culture française.

\section{Un parti pris pour les Canadiens français}

Le voyage au Canada de Tocqueville et de Beaumont a pris, dès le passage de la frontière en bateau à vapeur, une 
pente aventureuse, celle des surprises et des découvertes. Les rencontres intéressantes qu'ils ont d'abord faites, celles des Bois-Brûlés et des coureurs des bois, puis celles de Neilson, des frères Mondelet et des paysans canadiens, les ont d'abord menés à faire une excursion dans un musée imaginaire, celui de la vieille France. Ils ont ensuite été conduits dans une réflexion au long cours, concernant l'abandon de la colonie canadienne par la France, lors du « honteux traité de 1763, l'une des plus grandes ignominies de l'ignominieux règne de Louis $\mathrm{XV}^{40}$ ", comme Tocqueville l'écrivait à son frère, à bord du Fourth of July, en novembre 1831. Peut-être a-t-il éprouvé, comme un certain nombre de ses pairs voyageant au Canada, et notamment Châteaubriand avant lui, une espèce de " honte nationale » envers le "peuple sacrifié » des Canadiens français. C'est peut-être pourquoi, comme nous venons de le voir, il aurait borné ses observations au paradigme du Même:

En regardant sur les vieilles cartes l'étendue des anciennes colonies françaises en Amérique, je me demandais comment le gouvernement de mon pays avait pu laisser périr ces colonies, qui seraient aujourd'hui pour nous une source inépuisable de prospéritét ${ }^{41}$.

Les coenquêteurs n'ont cesse de s'interroger sur le destin collectif des Canadiens français, qu'ils considèrent comme formant une nation distincte en proie à une menace insidieuse : «les Canadiens n'ont pas cessé de former une population à part, entièrement distincte de la population anglaise qui cherche à s'introduire parmi eux ${ }^{42} \%$. Il est probable, écrit Dion, que «le caractère national français ait été chez Tocqueville une idée préconçue, antérieure à son voyage en Amérique. Mais le spectacle des relations anglo-canadiennes l'a visiblement conforté dans ses opinions ${ }^{43} »$. En effet, Tocqueville écrivait déjà à sa mère le 19 juin 1831, en prévision de son voyage au Bas-Canada : «Le Canada pique vivement notre 
curiosité. La nation française s'y est conservée intacte : on y a les mœurs et on y parle la langue du siècle de Louis $\mathrm{XIV}^{44}$.» On peut penser que Tocqueville s'est inspiré de cette expérience sensible lorsqu'il écrit, dans la Démocratie en Amérique: « il n'y a au monde que le patriotisme, ou la religion, qui puisse faire marcher pendant longtemps vers un même but l'universalité des citoyens ${ }^{45} \%$.

Tocqueville a l'œil alerte. Invité au cabinet de lecture par John Neilson, il dévore tous les journaux des derniers temps, anglais comme français. S'il trouve la langue française écrite médiocre, des tournures anglicisées et les idées sans grandeur $^{46}$, il perçoit néanmoins le sens du combat de la presse bas-canadienne :

Le Canadien a pour épigraphe: notre religion, notre langue, nos lois. Il est difficile d'être plus franc. Le contenu répond au titre. Tout ce qui peut enflammer les grandes et les petites passions populaires contre les Anglais est relevé avec soin dans le journal. J'ai vu un article dans lequel on disait que le Canada ne serait jamais heureux jusqu'à ce qu'il eût une administration canadienne de naissance, de principes, d'idées, de préjugés même, et que si le Canada échappait à l'Angleterre, ce ne serait pas pour devenir anglais.

L'éloignement physique de la population de la NouvelleFrance par rapport à la mère-patrie avait déjà motivé une appartenance du groupe d'origine française au territoire canadien et stimulé le développement d'une identité collective différente de celle des Français. Le passage de la colonisation française à l'occupation anglaise avait encore davantage décalé le rapport à la mère-patrie française jusqu'à la réduire à un lien imaginaire.

Ce sont les frères Mondelet qui ont « révélé » à Tocqueville et Beaumont « l'acuité du problème de la survivance française ». « Désormais, écrit Leclercq, le conflit ethnique est au 
centre de leurs préoccupations ${ }^{47}$.» En effet, si le questionnaire politique de Tocqueville prend forme, il ne se déploie que dans le cadre de la projection de rivalité ethnique :

Le clergé et une grande partie des classes non pas riches, mais éclairées, est [sic] français, ils commencent à sentir vivement leur position secondaire. Les journaux français que j'ai lus font contre les Français une opposition constante et animée. [...] Je ne puis croire qu'ils [Canadiens et Anglais] ne se fondent jamais, ni qu'il puisse exister une union indissoluble entre eux. J'espère encore que les Français, en dépit de la conquête, arriveront un jour à former à eux seuls un bel empire dans le Nouveau Monde, plus éclairés peut-être, plus moraux et plus heureux que leurs pères ${ }^{48}$.

S'agit-il pour Tocqueville, comme l'affirme Dion, "d'une forte réaction nationaliste et [d'] une nostalgie d'aristocrate ${ }^{49}$ " qui remettrait en marche la machine du rêve de l'Amérique française ? Il serait peut-être davantage à propos de voir dans les remarques de Tocqueville l'enthousiasme d'une découverte historique qui stimule l'imaginaire du jeune penseur et le fait réagir aux différents scénarios non-advenus de l'histoire coloniale canadienne et de l'histoire de la France de Napoléon.

De l'usage initial du nom Anglais, il lui substitue progressivement, dans le questionnaire adressé à ses interlocuteurs choisis, l'expression "peuple conquérant » et ensuite, le terme «ennemi»:

Je viens de voir dans le Bas-Canada un million de Français, braves, intelligents, faits pour former une grande nation française en Amérique, qui vivent en quelque sorte en étrangers dans leur pays. Le peuple conquérant tient le commerce, les emplois, la richesse, le pouvoir. Il forme les hautes classes et domine la société entière. Le peuple, conquis, partout où il n'a pas l'im- 
mense supériorité numérique perd peu à peu ses mœurs, sa langue, son caractère national ${ }^{50}$.

Précédé d'un an dans les villes de Québec et de Montréal par le jeune Théodore Pavie, qui publiera en 1833 ses Souvenirs atlantiques ${ }^{51}$, Tocqueville formule clairement une question nouvelle qui ne se lisait qu'en filigrane chez son compatriote : qu'en est-il des Français du Canada ? Ont-ils été absorbés par le conquérant? Sous la plume de Tocqueville, l'interrogation est franche et directrice : "Comment les Français au Canada souffrent-ils de la domination anglaise?" Il se nourrit alors des réponses concordantes pour élaborer un tableau dramatique de la situation politique et sociale du BasCanada :

Les Anglais jusqu'à présent se sont toujours tenus à part. Ils soutiennent le gouvernement contre la masse du peuple [...] Il y a donc fort à parier que le BasCanada finira par devenir un peuple entièrement français. Mais ça ne sera jamais un peuple nombreux. Tout deviendra anglais autour de lui. Ce sera une goutte d'eau dans l'océan ${ }^{52}$.

Les mots qu'il emploie afin d'animer les entretiens, pour désigner les Canadiens anglais et cerner les causes du déséquilibre des conditions politiques, économiques et sociales des deux groupes en présence, sont révélateurs de son engagement :

Plusieurs [Canadiens français] ne nous ont pas paru éloignés de se fondre avec les Anglais, si ceux-ci voulaient adopter les intérêts du pays. Il est donc à craindre qu'avec le temps et surtout l'émigration des Irlandais catholiques, la fusion ne s'opère. Et elle ne peut s'opérer qu'au détriment de la race, de la langue et des mœurs françaises ${ }^{53}$. 
À force de souligner, dans leurs commentaires et dans leurs questionnaires, le décalage entre les deux peuples et le souhait de voir les Canadiens se réapproprier complètement leur nationalité, peut-on avancer que Tocqueville et Beaumont sont pour autant des provocateurs ou des fomentateurs de révolution, comme le pense Pierson ${ }^{54}$ ? Suivant l'expérience révolutionnaire française en Amérique, aurait-il fallu se méfier de ces jeunes Français en territoire américain?

Nous arrivons au moment de la crise. Si les Canadiens ne sortent pas de leur apathie d'ici à vingt ans, il ne sera plus temps d'en sortir. Tout annonce que le réveil de ce peuple approche. Mais si dans cet effort les classes intermédiaires et supérieures de la population canadienne abandonnent les basses classes et se laissent entraîner dans le mouvement anglais, la race française est perdue en Amérique. Et ce serait en vérité dommage car il y a ici tous les éléments d'un grand peuple ${ }^{55}$.

Ces propos de Tocqueville ne sont que des souhaits. Au mieux, ils sont des observations lucides dont pourrait tirer parti un John Neilson. Les réponses aux questions de Tocqueville concernant la « libération du joug britannique » et la "domination anglaise " convergent en effet dans les propos d'un homme "à l'esprit vif et original ${ }^{56}$ ». John Neilson résume : "Nous avons déjà obtenu le redressement de quelques griefs. Quelques-uns uns existent encore. Mais il faudra bien que le gouvernement anglais fasse ce que nous voulons, il n'oserait pas nous refuser ${ }^{57}$ ». Aussi, le peuple canadien « en impose »-t-il aux Britanniques. En 1823, les Canadiens français ont dépêché Louis-Joseph Papineau et John Neilson afin de présenter une pétition de 60000 noms contre le projet d'union. à la suite de leur intervention, le gouvernement britannique renonça au projet d'union pour un temps, bien que " qu'il le considère toujours convenable et sage en théorie ${ }^{58}$ ". Résolu sans être résigné, John Neilson a le sentiment que : 
[...] les deux races vivront et se mêleront sur le même sol et que l'anglais restera la langue officielle des affaires. L'Amérique du Nord sera anglaise, la fortune a prononcé. Mais la race française du Canada ne disparaîtra pas. L'amalgame n'est pas aussi difficile à faire que vous pensez $z^{59}$.

«À quel point les effets sont ignorés de ceux qui font les causes ", répond en ce sens Monsieur Duponceau à Tocqueville, un "vieillard estimé », Français d'origine et habitant les États-Unis depuis plus de soixante ans. Il poursuit en disant: "Je ne doute pas que si l'Angleterre n'eût pas conquis le Canada en 1763, la Révolution américaine n'eût pas eu lieu. Nous serions encore Anglais ${ }^{60} »$. Tocqueville semble en effet prendre parti pour l'avenir des Canadiens plus que ne le ferait un observateur objectif. Aussi considère-t-il comme un péril pour la nation canadienne-française la montée d'une classe moyenne libérale en son sein, largement représentée dans la propriété des organes de presse ${ }^{61}$. Il pose ainsi un regard méfiant sur une classe de personnes à l'appartenance ambivalente. Ils sont Canadiens mais plus tout à fait Français ou carrément des Anglais...

Il existe déjà à Québec une classe d'hommes qui forme la transition entre le Français et l'Anglais : ce sont des Anglais alliés à des Canadiens, des Anglais mécontents de l'administration, des Français en place. Cette classe est représentée dans la presse périodique par la Gazette de Québec, mélange de français et d'anglais, dans les assemblées politiques par M. Neilson et probablement plusieurs autres que nous ne connaissons pas. C'est elle que je crains le plus pour le sort futur de la population canadienne. Elle n'excite ni sa jalousie, ni ses passions. Au contraire elle est plus canadienne qu'anglaise d'intérêt parce qu'elle fait de l'opposition au gouvernement. Au fond, cependant, elle est anglaise de mœurs, d'idées, de langue. Si elle prenait jamais la place des hautes clas- 
ses et des classes éclairées parmi les Canadiens, la nationalité de ceux-ci serait perdue sans retour. Ils végéteraient comme les bas-Bretons en France ${ }^{62}$.

Le jeune fonctionnaire français voit en effet d'un mauvais œil la légendaire tolérance britannique, qui risque d'endormir les aspirations politiques des Canadiens français :

Au total, cette population nous a paru capable d'être dirigée quoique encore incapable de se diriger ellemême. Nous arrivons au moment de la crise. Si les Canadiens ne sortent pas de leur apathie d'ici à vingt ans, il ne sera plus temps d'en sortir. Tout annonce que l'éveil de ce peuple approche.

Selon Tocqueville, l'attitude des Canadiens français serait peut-être héritière d'un trait national français, acquis par l'habitude d'être sous la tutelle de son gouvernement: «Les paysans se souviennent de l'état de sujétion dans lequel on les tenait sous le gouvernement français ${ }^{63} »$. Encore une fois, John Neilson confirme : "Le Canadien est tendrement attaché au sol qui l'a vu naître, à son clocher, à sa famille. C'est ce qui fait qu'il est si difficile de l'engager à aller chercher fortune ailleurs ». Le Canadien a alors peu de goût pour l'indépendance. L'intangibilité de cette aspiration serait alors seule responsable du faible essor de la colonie de la Nouvelle-France - en comparaison aux treize colonies britanniques devenues les États-Unis - et de la faible résistance à la menace d'assimilation ${ }^{64}$.

Encore ému de la révolution de 1830, à laquelle il pense en 1831, perturbé par le brouillard et la forêt dense qui lui rappellent les fumées des canons, Tocqueville n'a rien d'un agitateur. Nul doute qu'il aura pris le pouls du mécontentement populaire des Canadiens à l'endroit du gouvernement britannique et de la situation précaire de la survivance du fait français en Amérique. Tocqueville n'hésite pas à prendre parti 
pour les Canadiens afin de creuser davantage l'opinion générale sur un projet de société en réelle genèse qui allait, pendant l'année 1837, éclater en rébellion :

À l'époque de mon passage, écrit Tocqueville en 1838, les Canadiens étaient pleins de préjugés contre les Anglais qui habitaient au milieu d'eux, mais ils semblaient sincèrement attachés au gouvernement anglais qu'ils regardaient comme un arbitre désintéressé placé entre eux et cette population anglaise qu'ils redoutaient. Comment est-il arrivé qu'ils soient devenus les ennemis de ce même gouvernement ${ }^{65}$ ?

\section{$* * *$}

Le séjour de Tocqueville au Bas-Canada rencontre encore aujourd'hui beaucoup d'intérêt de la part des historiens et des politologues québécois puisqu'il survient à un moment creux dans les relations entre la Nouvelle-France et sa mèrepatrie : «Entre le traité de Paris de 1763 et la visite de La Capricieuse en 1855, il [le voyage de Tocqueville au Canada] fut le seul fait majeur des relations culturelles franco-canadiennes ${ }^{66}$. $)$ Aussi, en 1987, l'historien Heinz Weinmann, commentant la première défaite référendaire des tenants de l'indépendance du Québec, écrivait-il : «Les événements politiques depuis 1980 donnent raison à Tocqueville qui, voilà plus de 150 ans, affirmait que le "Français se sent peu de goût pour l'indépendance ${ }^{67} »$. Tout comme Tocqueville, pour qui la cohérence et la constance des questions posées à l'histoire en marche étaient plus importantes que les présupposés, différents partis politiques aux visées opposées vont tour à tour citer Tocqueville pour fonder l'historicité et l'autorité de leur argumentation.

On ne saurait conclure sur l'importance des réflexions de Tocqueville concernant le Bas-Canada à la manière de 
Dion, qui assure que les notes éparses du jeune voyageur « annoncent les thèses que l'homme mûr développera sur l'Ancien Régime, la révolution et la démocratie ${ }^{68}$ » ou de Corbo qui fait de l'auteur de la Démocratie en Amérique un visionnaire de la "situation durable» des Canadiens français :

La croissance démographique soutenue de la population de langue anglaise en Amérique du Nord pénètre jusque dans les rangs des anciens maitres $d u$ sol, domine leurs villes et dénature leur langue. Ainsi, Tocqueville annonce le destin que vivront durablement les Français du BasCanada ${ }^{69}$.

À dessiner des parallèles, des oppositions ou des analogies entre les colonies françaises et britanniques de l'Amérique, on peut en revanche se demander pourquoi Tocqueville résiste à évoquer, même subrepticement, la question de l'américanité des Canadiens. Si l'Amérique appartient à l'avenir et la population canadienne au passé de la France, pourquoi Tocqueville ne suppose-t-il pas le caractère d'américanité, et donc de nouveauté, de la société bas-canadienne?

Le jeune voyageur se découvrant lui-même aura vu s'épanouir la dimension imaginaire de son identité, c'est-àdire la vieille France dans un Nouveau Monde américain. Nous sommes davantage de l'avis de Corbo lorsqu'il résume : «En substance, on peut dire que, pour Tocqueville, le Bas-Canada a constitué une découverte ou une révélation à la fois émouvante et douloureuse ${ }^{70}$. » Le premier tiers du XIX ${ }^{e}$ siècle bascanadien était en fait le terrain mentalitaire idéal pour poser la question de la survie et de la proclamation de l'identité au sein d'une structure qui aurait pris en considération sa portion continentale. Confiné, par la force de son imaginaire national encore attaché aux représentations de la vieille France en Nouvelle-France devenue Bas-Canada, borné, par son expérience de Français en Amérique, à penser sa relation aux 
Canadiens sur le territoire identitaire du Même, le jeune Alexis de Tocqueville ne pouvait pas être un motivateur de la rébellion des Patriotes puisque celle-ci, dans son préambule, contenait en germe cette double distinction: les Canadiens ne seraient ni Anglais, ni Français. En effet, la difficile question de la rénovation de l'héritage français et de la tradition britannique en territoire bas-canadien relevait davantage de la refondation identitaire que du rapatriement d'une identité telle qu'imaginée par Tocqueville. Ainsi, bien que ce processus de redéfinition nationale fut perçu comme salutaire par Tocqueville et même, dans une certaine mesure, soutenu par lui, Tocqueville n'a peut-être pas pu voir ce mouvement imperceptible à l'œil du voyageur en quête du Même, c'est-à-dire le simple espoir de dépassement d'une ambivalence identitaire.

\section{NOTES}

'Selon les sources et le croisement des dates évoquées par Gustave de Beaumont et Alexis de Tocqueville, de même qu'à l'aide du tableau synoptique esquissé par Jean-Michel Leclercq, auteur d'un mémoire de DES sur le sujet (Lille, 1965) et d'un article précieux (" Alexis de Tocqueville au Canada (du 24 août au 2 septembre 1831) ", Revue d'bistoire de l'Amérique française, vol. 22, n" 3 (1968), pp. 353-364), il nous est possible de tracer le parcours que Tocqueville et Beaumont suivent au Bas-Canada ainsi que les moments de l'enquête qu'ils y mènent à la fin de l'été 1831. Le 24 août 1831, ils sont à Montréal où ils rencontrent le père Quiblier, supérieur du Séminaire, et les deux frères Dominique et Charles Mondelet, avocats. Le 25 août, ils voguent sur le SaintLaurent et rédigent une fiche sur l'apparence physique du Canada. Le 26 août, à Québec, ils s'entretiennent avec Nomen Blanc, « un négociant anglais », et assistent à une plaidoirie à un tribunal civil de Québec. Le 27 août, ils questionnent John Neilson, député à la Chambre d'Assemblée, et le suivent pour une visite au Cabinet de lecture où ils découvrent les journaux du Haut et du Bas-Canada. Les trois jours suivants sont faits de balades « en solitaire » ou en compagnie de John Neilson et de Denis-Benjamin Viger (Tocqueville écrit Niger et n'est plus certain de son nom). Le $1^{\mathrm{cr}}$ septembre, Tocqueville écrit ses 
«Remarques générales » sur le Canada et enfin, le 2 septembre, les deux amis rencontrent le juge Jean-Thomas Taschereau.

${ }^{2} \mathrm{Au}$ cours de ce texte, nous employons ce néologisme comme une épithète, remplaçant précisément le groupe complément du nom "de la mentalité » qui serait grammaticalement plus juste mais moins imagé et plus lourd. Nous demandons au lecteur de bien vouloir en accepter l'usage, comme il le ferait pour les textes de Jocelyn Létourneau.

${ }^{3}$ Il va sans dire que la question de l'identité est le concept moteur et directeur de la narration. Voir, sur l'identité du sujet narratif en histoire, entre autres ouvrages de Paul Ricœur, La mémoire, l'bistoire, l'oubli, Paris, Seuil, 2000, 675 p. (Coll. «L'Ordre philosophique »).

${ }^{4}$ Puisque Tocqueville signe la majorité des documents écrits relatifs au voyage au Bas-Canada, même si Beaumont y a nécessairement mis la main, nous nous référons à Tocqueville sauf mention explicite de l'extrait retrouvé du journal ou de la correspondance personnelle de Beaumont. Mentionnons d'emblée le remarquable ouvrage de compilation qu'a réalisé Claude Corbo même si nous n'y faisons que peu référence, compte tenu de la simultanéité de nos recherches pendant les années 2002 et 2003 (voir Alexis de Tocqueville, Regards sur le Bas-Canada, choix de textes et présentation de Claude Corbo, Montréal, Typo, 2003, 322 p.). Il s'agit d'un complément essentiel à l'ouvrage de Jacques Vallée (Tocqueville au Bas-Canada, Montréal, Éditions du Jour, 1973, 187 p.) puisque l'édition des Euvres complètes de Tocqueville s'est poursuivie de 1951 à 2004. Les présentations de textes sont pertinentes et le contexte politique et géographique du Bas-Canada est clairement défini. Cet ouvrage, qui s'avère très utile à la recherche universitaire sur Tocqueville au Bas-Canada, est essentiellement le rassemblement de textes autrement éparpillés dans différents ouvrages, pensons à George Wilson Pierson, Tocqueville in America, Baltimore et Londres, Johns Hopkins Paperbacks Edition, 1996 [1938], 852 p., André Jardin, Alexis de Tocqueville, Paris, Hachette, 1984, 522 p., Alexis de Tocqueville, CEuvres complètes, Paris, La Pléiade, 18 tomes et 30 volumes, en cours de publication depuis 1951. Corbo nous allège la tâche en présentant même les rares fragments du journal de Gustave de Beaumont et sa correspondance relative au voyage au Bas-Canada (Gustave de Beaumont, Lettres d'Amériques : 1831-1832, Paris, Presses Universitaires de France, 1973, 220 p.)

${ }^{5}$ René Rémond, Les États-Unis devant l'opinion française (1815-1852), Paris, Armand Colin, 1962, p. 379, cité par Gérard Bergeron, Quand Tocqueville et Siegried nous observaient, Sillery, Presse de l'Université du Québec, 1990, p. 60. 
${ }^{6}$ Ils sont soit numérotés, soit thématiques et fonctionnent pour la plupart selon un classement intérieur par ordre alphabétique de noms propres et de noms communs intercalés, identifiant à la fois des lieux, des personnes ou des concepts. Ces cahiers, dont les thèmes s'entremêlent parfois, sous les entrées «Démocratie » ou "Canada », pour ne prendre que ces exemples, sont eux-mêmes dispersés dans les différents tomes et volumes des CEuvres completes de Tocqueville, y compris dans sa correspondance.

${ }^{7}$ Toute la documentation scientifique et spécialisée que nous avons parcourue concernant le voyage de Tocqueville au Canada est tributaire de la biographie écrite par André Jardin, op. cit.

${ }^{8}$ Corbo, op. cit., p. 38.

"Selon nombre d'historiens dont les critiques sont résumées dans une note écrite par Stéphane Dion, «Tocqueville surestime le niveau de vie et la proportion de francophones, sous-estime l'importance des villes et le poids des charges seigneuriales, connaît mal les problèmes politiques et parait même ignorer l'existence de Louis-Joseph Papineau, mais prévoit la survie des Canadiens français [...] ». «La pensée de Tocqueville - l'épreuve du Canada français ", Revue d'bistoire de l'Amérique française, vol. 41, n" 4 (1988), p. 538.

${ }^{10}$ Entretien réalisé à Québec, le 26 août 1831 : Tocqueville, Cahier non-alphabétique I, à l'entrée « voyage en Amérique », Euvres Complètes, tome 5, vol. 1, 1957, p. 79.

"Ibid., p. 81.

${ }^{12}$ En effet, « [...] pour l'essentiel, Neilson apparait comme un des premiers idéaltypes du "Canadien" au sens contemporain : bilingue, lié à des personnes de diverses origines et croyant en l'avenir du pays. Son échec relatif et son incapacité à se créer des réseaux de liens solides et durables en milieu canadienfrançais (en affaires et en politique) témoignent de la difficulté de l'entreprise à toute époque ", écrivent Sonia Chassé, Rita Girard-Wallot et Jean-Pierre Wallot, "Neilson, John", Dictionnaire biographique du Canada, www.biographi.ca/fr/.

${ }^{13}$ Le Parti canadien est devenu le Parti patriote en 1826.

${ }^{1+}$ Tocqueville, Cahier alphabétique A, Euvres complètes, tome V, vol. 1, pp. 212 et 215 .

${ }^{15}$ Spencer invite les deux amis à séjourner dans sa maison d'été, à Canandaigua. C'est dans le cadre de vacances qu'il explique à Tocqueville les différences entre les systèmes judiciaires français et américain et gagne ainsi sa confiance et son 
estime. Il deviendra, plus tard, un participant à l'élaboration de la traduction De la démocratie en Amérique. Democracy in America, traduction de Henry Reeve, préface et notes de John Canfield Spencer, New York, Adlard and Saunders, 1838, 464 p. Pour une description détaillée de ces événements, voir George Wilson Pierson, Tocqueville in America, Baltimore et Londres, Johns Hopkins Paperbacks Edition, 1996 [1938], 852 p.

${ }^{16}$ Tocqueville, CEuvres complètes, tome V, vol. 1, p. 214.

${ }^{17}$ Leclercq, loc. cit., p. 362.

${ }^{18}$ Dion, loc. cit., p. 542.

${ }^{19}$ Corbo, op. cit., p. 182 fait référence aux Euvres complètes, tome V, vol. 1, pp. 213-214 de même que la citation suivante, qui se trouve être la clôture de son raisonnement.

${ }^{20}$ Pierre Pouchot, Mémoires sur la dernière guerre de l'Amérique du Nord, [Yverdon, 1781], cité par Armand Yon, « Les Canadiens français jugés par les Français de France 1830-1939", Revue d'bistoire de l'Amérique française, vol. 18, n’3 3 (196465), p. 321. Voir la réédition: Pierre Pouchot, Mémoires de la dernière guerre de l'Amérique septentrionale, Septentrion, Sillery (Québec), 2003, 324 p.

${ }^{21}$ Alexis de Tocqueville dans une lettre adressée à l'Abbé Lesueur, cité par Jacques Vallée, Tocqueville an Bas-Canada, Montréal, Éditions du Jour, 1973, p. 107 et Corbo, op. cit., p. 37.

22 Atala-René fut publié à part en 1807 à Paris.

${ }^{23}$ Bergeron, op. cit., p. 71.

${ }^{24}$ Tocqueville, Cahier non-alphabétique, Euvres complètes, tome V, vol. 1, p. 174.

${ }^{25}$ Tocqueville, Cahier alphabétique à l'article « Canada », réflexion datée du 25 août 1831, CEurres complètes, tome V, vol. 1, p. 210.

${ }^{26}$ Corbo, op. cit., p. 9.

${ }^{27}$ Tocqueville, CEurres completes, tome V, vol. I, p. 84.

${ }^{28}$ L'expression est présente, entre autres, chez Jacques Madaule, Les chrétiens dans la cité, Paris, Sagittaire, 1946.

29) Tocqueville, Cahier non-alphabétique, Euvres complètes, tome V, vol. I, p. 174. 
${ }^{30}$ Lettre d'Alexis de Tocqueville à son père recensée par Jacques Vallée, op. cit., p. 81 .

31 Tocqueville, CEuvres complètes, tome 7, p. 87.

32 Ibid., tome V, vol. I, p. 215.

${ }^{33}$ Note à la suite de l'entretien avec John Neilson, Ibid., p. 84.

${ }^{3+}$ Cahier alphabétique à l'article « Canada », Ibid., p. 210.

35 Ibid., p. 211.

"36 Idem, L'Ancien Régime et la Révolution, Paris, Flammarion, 1985, p. 320. (Coll. «Folio »).

${ }^{37}$ Idem, 25 novembre 1831, cahier portatif numéro 3, Euvres complètes, tome V, vol. I, p. 189.

${ }^{38}$ Lettre inédite communiquée par Henri Chardon au journal Le Canada (18 juin 1935) citée par Armand Yon, [destinataire de la lettre non-mentionné] loc. cit., p. 333.

3) Lettre à l'abbé Lesueur datée d'Albany, le 7 septembre 1831, citée par Jacques Vallée, op. cit., p. 107 et citée par Corbo, op. cit., p. 37 en référant à la Correspondance familiale, Euvres complètes, tome XV, 1998, p. 129.

${ }^{40}$ Lettre de Tocqueville à son frère recensée par Vallée, op .cit., p. 114.

${ }^{+1}$ Châteaubriand, Mémoires d'outre-tombe, Paris, Garnier, 1859, tome 1, p. 393.

+2 Jardin cite Gustave de Beaumont, op. cit., p. 139.

t3 Dion, loc. cit., p. 542.

${ }^{H}$ Tocqueville cité par Corbo, op. cit., p. 37 en référant aux CEuvres complètes, vol. $\mathrm{XV}, \mathrm{p} .105$. On peut en effet voir une contradiction dans ce propos, puisque dans sa lettre à l'abbé Lesueur du 7 septembre 1831 (note 20), Tocqueville fait un bilan de son voyage et écrit «Il n'y a pas six mois, je croyais, comme tout le monde, que le Canada était devenu complètement anglais. J'en étais toujours resté au relevé de 1763, qui n'en portait la population française qu'à 60 000 personnes $»$. En revanche, dans son commentaire à sa mère, il parle de ce qu'il lui a été annoncé, et ce, seulement deux mois avant son voyage, c'est-àdire alors qu'il était déjà aux États-Unis. Avant son arrivée en Amérique, il aurait pu penser en effet, comme le dit à son ami Lesueur, que " personne » ne parlait plus le français au Canada. C'est donc en faisant son bilan qu'il se remémore son « ignorance » du fait français, laquelle il exagère peut-être, pour paraître meilleur élève ? Pour parer à cette contradiction remarquée par notre 
évaluateur, il faudrait ici étudier la rhétorique interne à la correspondance de Tocqueville qui, à sa mère, écrit qu'il sait et à son ancien précepteur, qu'il a appris.

${ }^{45}$ Tocqueville, CEuvres complètes, tome I, chapitre 10, p. 535. À propos de la religion, le clergé catholique du Canada a droit à un jugement de valeur positif chez Tocqueville : « Point d'ornements bizarres et ridicules, point d'ex-votos dans les églises. La religion [est] éclairée et le catholicisme ici n'excite ni à la haine ni au sarcasme des protestants. J'avoue que pour ma part, elle satisfait plus à mon esprit que le protestantisme des États-Unis. Le curé est bien véritablement ici le pasteur du troupeau ; ce n'est point un entrepreneur d'industrie religieuse comme la plupart des ministres américains. Ou il faut nier l'utilité d'un clergé, ou il faut l'avoir comme au Canada » écrit Tocqueville, Ibid., tome V, vol. I, p. 211. Le lecteur trouvera un commentaire substantiel du lien entre la citoyenneté et la religion chez Tocqueville en consultant la thèse de doctorat de Agnès Antoine, L'Impensé de la démocratie : Tocqueville, la citoyenneté et la religion, Paris, Fayard, 2003.

${ }^{46}$ «Les gazettes du Canada [...]Elle [la langue] a un tour simple et naif fort éloigné de nos grands mots, de l'emphase et de la simplicité affectée de notre littérature actuelle, mais elle roule sur de petites ou de vieilles idées » écrit Tocqueville dans son cahier alphabétique A, sous la rubrique "Canada », CEwres complètes, tome V, vol. I, p. 216 de même que la citation suivante.

${ }^{47}$ Leclerq, loc. cit., p. 363.

${ }^{48}$ Premières notes sur le Canada, le 25 août 1831, CEurres complètes, tome V, vol. I, pp. 77-78.

${ }^{49}$ Dion, loc. cit., p. 537.

${ }^{50}$ Corbo, op. cit., pp. 211-212 fait référence aux CEuvres complètes, vol. XIV, pp. 145-147.

${ }^{51}$ Théodore Pavie, Souvenirs atlantiques, Paris, Bossange, 1833, 2 volumes.

${ }^{52}$ Tocqueville, Notes chiffrées 2 et 6, Euvres complètes, tome V, vol. I, pp. 217 et 218 .

${ }^{53} 1^{\text {cr }}$ septembre 1831 [le 3 selon Beaumont] Tocqueville fait encore d'autres remarques. Le passage est tiré de Ibid., p. 217.

${ }^{54}$ Pierson écrit : «The prison commissioners had come out from Quebec without Mr. Neilson precisely so that they could discuss the future of Lower Canada with the Norman peasants, perhaps even to suggest to them a racial policy, at least discover whether it was within the range of possibility to 
arouse them to a struggle for independence and greatness. [...] In short, for the moment the two investigators had cast discretion to the winds, and were acting the role of instigators of revolution. There was no other name for what they were doing » Pierson, p. 339 cité par Bergeron, op. cit., p. 76.

${ }^{55}$ Note du 29 août 1831 citée par Corbo, op. cit., p. 182 en référant aux CEuvres complètes, vol. 5, pp. 213-214.

${ }^{56}$ CEuvres complètes, tome V, vol. I, p. 80.

${ }^{57}$ Bergeron, op. cit., p. 63 cite un fragment du journal de Gustave de Beaumont [source exacte non-citée].

${ }^{58}$ Yvan Lamonde cite Documents relatifs à l'histoire constitutionnelle du Canada II, op. cit., p. 148 en p. 94.

${ }^{59}$ Euvres complètes, tome V, vol. I, p. 81.

${ }^{60}$ Cahiers non-alphabétiques 2 et 3, Ibid., p. 106.

${ }^{61}$ «Il paraît à Québec un journal appelé La Gazette, semi-anglais, semi-français ; et un journal absolument français appelé Le Canadien. Ces journaux ont à peu près la dimension de nos journaux français [...] ils font une opposition violente au gouvernement et même à tout ce qui est anglais. " Tocqueville, Cahier alphabétique A, Ibid., pp. 211-212.

62 Tocqueville, Cahier alphabétique A, Ibid., p. 215, de même que la citation suivante.

${ }^{63}$ Ibid., p. 82, de même que la citation suivante.

${ }^{6+}$ Ce type de réflexion sourd d'une explication complémentaire à ses notes de voyages sur le Bas-Canada, consignées dans un court chapitre intitulé : Quelques idées sur les raisons qui s'opposent à ce que les Français aient de bonnes colonies. Quelques années plus tard, il ajoutera : "Quand je veux juger l'esprit de l'administration de Louis XIV et ses vices, c'est au Canada que je dois aller. On aperçoit alors la difformité de l'objet comme dans un microscope. » Tocqueville, L'Ancien Régime et la Révolution, op. cit., p. 351.

${ }^{65}$ Alexis de Tocqueville à Henry Reeve, clerc du conseil privé, le 3 janvier 1838 , archives de l'Université de Toronto ; Canadian Historical Review, vol. XIX (1938), pp. 394-397.

${ }^{61}$ Bergeron, op. cit., p. 80.

${ }^{67}$ Heinz Weinmann, Du Canada au Québec : généalogie d'une histoire, Montréal, L'Hexagone, 1987, p. 235. 
${ }^{68}$ Dion, loc. cit., p. 539.

(9) Corbo cite d'abord le deuxième tome de la Démocratie en Amérique (1840) puis fait ce commentaire, op. cit., p. 248.

${ }^{70}$ Ibid., p. 40. 